Check for updates

Cite this: Chem. Sci., 2019, 10, 10629

๑ All publication charges for this article have been paid for by the Royal Society of Chemistry

Received 6th August 2019

Accepted 26th September 2019

DOI: $10.1039 / c 9 s c 03913 a$

rsc.li/chemical-science

\section{Tuning symmetry breaking charge separation in perylene bichromophores by conformational control $\uparrow$}

\author{
Alexander Aster, (ID) a Giuseppe Licari, (iD ${ }^{c d}$ Francesco Zinna, (D) ${ }^{\mathrm{b}}$ Elodie Brun, (DD ${ }^{\mathrm{b}}$ \\ Tatu Kumpulainen, (D) a Emad Tajkhorshid, (D) cd Jérôme Lacour (iD b \\ and Eric Vauthey $(\mathbb{D}$ *a
}

Understanding structure-property relationships in multichromophoric molecular architectures is a crucial step in establishing new design principles in organic electronics as well as to fully understand how nature exploits solar energy. Here, we study the excited state dynamics of three bichromophores consisting of two perylene chromophores linked to three different crown-ether backbones, using stationary and ultrafast electronic spectroscopy combined with molecular dynamics simulations. The conformational space available to the bichromophores depends on the structure and geometry of the crown-ether and can be significantly changed upon cation binding, strongly affecting the excited-state dynamics. We show that, depending on the conformational restrictions and the local environment, the nature of the excited state varies greatly, going from an excimer to a symmetry-broken charge separated state. These results can be rationalised in terms of a structure-property relationship that includes the effect of the local environment.

\section{Introduction}

Multichromophoric systems play important roles in biology as well as in technological applications, because they can efficiently absorb light, funnel the energy toward a defined location and separate electric charges. ${ }^{\mathbf{1 - 1 6}}$ Specific optimisation of a material for one or several of these processes can be achieved by tuning the properties and the spatial arrangement of the chromophoric sub-units. ${ }^{17-26}$

The simplest multichromophoric array, comprising two identical sub-units, can be used to model the impact of geometry on material properties. These so-called bichromophores are particularly useful in investigating the mixing of the isoenergetic diabatic electronic states of the monomers as they approach each other. ${ }^{27-33}$

\footnotetext{
${ }^{a}$ Department of Physical Chemistry, University of Geneva, CH-1211 Geneva, Switzerland. E-mail: eric.vauthey@unige.ch

${ }^{b}$ Department of Organic Chemistry, University of Geneva, CH-1211 Geneva, Switzerland

${ }^{c}$ NIH Center for Macromolecular Modeling and Bioinformatics, Beckman Institute for Advanced Science and Technology, University of Illinois at Urbana-Champaign, Urbana, Illinois, USA

${ }^{d}$ Department of Biochemistry, Center for Biophysics and Quantitative Biology, Urbana, Illinois, USA

$\dagger$ Electronic supplementary information (ESI) available: Experimental details, additional spectroscopic data, additional MD simulations data, global analysis. See DOI: 10.1039/c9sc03913a. All experimental data can be downloaded from http://doi.org/10.5281/zenodo.3472622.
}

Since the Kasha/Davydov aggregate model in the 1960s introducing the Frenkel excitonic states for molecular dimers, ${ }^{29,31}$ substantial theoretical effort has been invested to understand the different states of such dimers and the effect of mutual orientation and distance. ${ }^{27,28,30}$ In the weak coupling limit, the dimer excited states can be theoretically described in terms of symmetry-broken local-excited (LE) states and charge-separated (CS) states. In the strong coupling limit, electronic excitation is distributed over the dimer, and the states can be described as coherent superpositions of the LE states, so-called Frenkel excitonic states, and of the CS states, resulting in charge-resonance states. In turn, Frenkel excitonic states and charge resonance states can mix to yield the experimentally observable states (Fig. S2†) ${ }^{30,34}$ In vacuum, these states are symmetric, but in a disordered environment, e.g. a liquid or a protein, local asymmetry can lead to an uneven distribution of the electronic density on the two sub-units. Therefore, a symmetric dimer with strong charge resonance character transforms into a symmetry-broken charge-transfer (CT) state with a substantial dipole moment. Depending on the local field asymmetry and on the molecular orbital overlap of the two sub-units, the CT character can be close to $100 \%$, corresponding to a CS state. Photoinduced symmetrybreaking (SB) charge separation (CS) \% could be particularly advantageous in materials for applications involving lightharvesting and charge transfer, because, first, the material can be based on a single type of chromophore and, second, the energy loss upon population of the CS state is minimal..$^{35-44}$ This process has been suggested to be triggered by the thermal fluctuations of 
the solvent field, which lift transiently the degeneracy of the two diabatic CS states. As solvent relaxation takes place, the energy splitting between these two states increases, and the system is trapped in the lowest states. ${ }^{\mathbf{4 2 , 4 3 , 4 5 , 4 6}}$

In addition to the environment, the mutual orientation and interchromophoric distance, which influence the coupling, can be expected to have a significant impact on SB. Unambiguous evidence of SB-CS between two identical molecules was obtained using a bichromophore comprising two perylene (Pe) moieties connected through a propyl linker (Pe-Pr-Pe). ${ }^{42}$ Unfortunately, because of the flexible linker, the Pe sub-units could adopt a wide range of mutual orientations and distances, hindering a detailed analysis of the structure-property relationship. More recently, several groups investigated $\pi$ stacked bichromophores, either covalently linked with stiff backbones or designed for self-assembly, and allowing for precise control of mutual geometry. ${ }^{47-51}$ These studies were mostly limited to closely $\pi$-stacked conformations and the differences in the dynamics and the nature of the excited states between the various structures were rather subtle.

Here, we combine the two approaches, first, to have control over the conformation and, second, to cover a wide range of distances and coupling energies. For this, we investigate a series of bichromophores consisting of two identical Pe heads connected through different crown-ether backbones (Fig. 1). ${ }^{52}$ Additionally to the various backbones, which already lead to distinct Pe-Pe distances and orientations, binding of a cation drastically changes the conformation of the macrocycle, ${ }^{52-56}$ and, thus, the orientational restrictions on the Pe heads. Spectroscopic studies are complemented with molecular dynamics (MD) simulations to estimate the most probable geometries adopted by the bichromophores at room temperature.

We begin with $18 \mathrm{C6}$ and its $\mathrm{Ba}^{2+}$ complex in acetonitrile, first discussing the stationary electronic spectroscopy and MD simulations results to evaluate the conformational space accessible for this dimer. Based on this, we discuss the excitedstate dynamics investigated using a combination of timeresolved techniques. These results are then compared with those obtained with 18C6, 18C4, and $16 \mathrm{C4}$ and well as their $\mathrm{Na}^{+}$

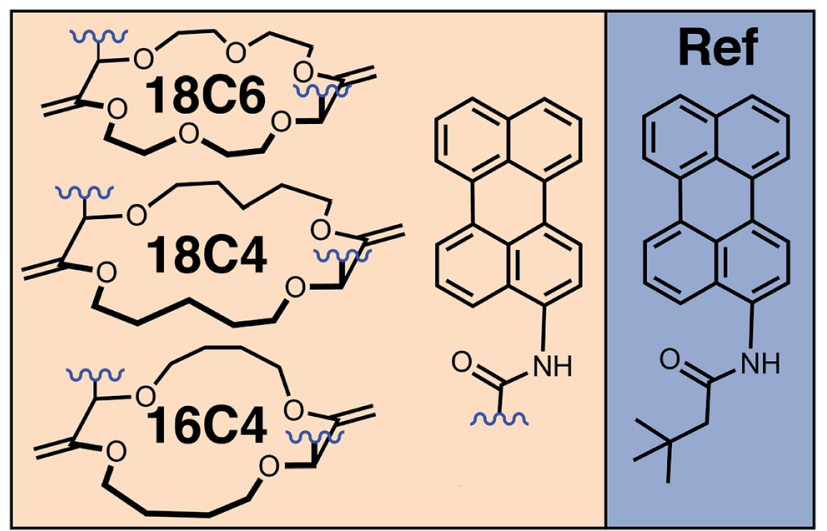

Fig. 1 Chemical structures of the perylene (Pe)-based crown-ether bichromophores and of the monomeric reference (Ref). complexes in dichloromethane to draw a comprehensive picture of the impact of conformation and local environment. We will show that the nature of the relaxed excited state is highly sensitive to these parameters, which can be used to tune the properties towards a high SB-CS yield.

\section{Experimental part}

The syntheses of 18C6, 18C4, 16C4 and Ref were previously described elsewhere. ${ }^{52}$ All details on the MD simulations as well as the time-resolved and stationary experiments can be found in the ESI. $\uparrow$ Femtosecond electronic transient absorption (TA) in the visible (VIS) and 800-1600 $\mathrm{nm}$ (NIR) regions and broadband fluorescence up-conversion spectroscopy (FLUPS) measurements were done upon $400 \mathrm{~nm}$ excitation, whereas the nanosecond TAVIS experiments were performed upon $355 \mathrm{~nm}$ excitation.

\section{Results and discussion}

\section{$3.118 \mathrm{C} 6$ and $18 \mathrm{C} 6 \subset \mathrm{Ba}^{2+}$}

3.1.1 Stationary spectra and MD simulations. The electronic absorption and emission spectra of 18C6 depicted in Fig. 2A point to a substantial coupling between the Pe chromophores. The intensity ratio of the $0-0$ and first vibronic absorption bands $\left(I_{0-0} / I_{1-0}\right)$ is inverted compared to the Ref
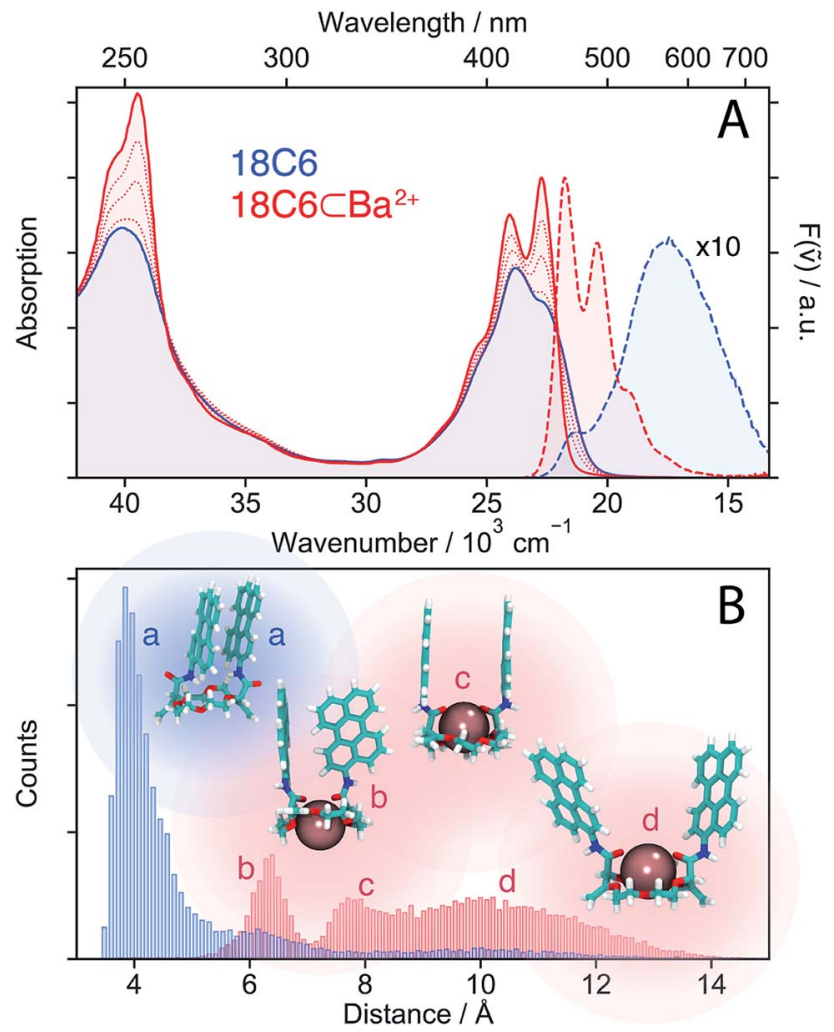

Fig. 2 (A) Stationary electronic absorption and emission spectra of $18 \mathrm{C} 6$ in acetonitrile (blue) and upon addition of $\mathrm{Ba}\left(\mathrm{ClO}_{4}\right)_{2}$ (red). (B) Histogram of centre-to-centre distances illustrating the conformational changes upon cation binding along with representative snapshots from the MD simulations. 
monomer (Fig. S5 $\dagger$ ). This is a typical signature of a strongly coupled dimer with H-type conformation. ${ }^{34}$ Moreover, the emission band is broad, structureless, exhibits a strong Stokes shift and resembles the fluorescence reported for the Pe excimer. ${ }^{57,58}$ Upon addition of $\mathrm{Ba}\left(\mathrm{ClO}_{4}\right)_{2}$, the relative intensity of the 0-0 transition increases and the vibronic structure becomes more pronounced, whereas the emission spectrum transforms into an intense band with mirror-image symmetry. These changes can be analysed assuming the binding of a single $\mathrm{Ba}^{2+}$ guest to the macrocycle host (18C6), giving $18 \mathrm{C6} \subset \mathbf{B a}^{2+}$ with an association constant of $K=4 \times 10^{7} \mathrm{M}^{-1}$ (Table S1, ESI $\dagger$ ).

The similarity of the spectra of $18 \mathrm{C6} \subset \mathbf{B a}^{2+}$ with those of Ref points to a weak coupling between the two chromophores and suggests that absorption and emission involve LE states. In the absence of $\mathrm{Ba}^{2+}$, emission occurs from a lower-energy state and has smaller oscillator strength than the LE emission, as expected for an excimer state.

MD simulations were performed to obtain a quantitative picture of the conformational space accessible to $18 \mathrm{C6}$ and $18 \mathrm{C6} \subset \mathrm{Ba}^{2+}$. Geometrical parameters, namely the Pe-Pe centreto-centre distance, the angle between the long axes of the Pe heads, the angle between the Pe planes and the slip angle, were extracted from the ensuing trajectories (see Fig. $\mathrm{S} 1 \uparrow$ for the definition). As depicted in Fig. 2B, the two Pe sub-units in $18 \mathrm{C6}$ are closely $\pi$-stacked, in agreement with the significant coupling inferred from the stationary spectra. The angle between the long axes of the Pe heads is distributed around $30^{\circ}$ (Fig. S9†), in line with previous quantum-chemical calculations predicting a ground-state minimum geometry of the Pe dimer with $D_{2}$ symmetry (Fig. S3 $\dagger$ ). ${ }^{30}$ This indicates that, in contrast to a stiff backbone, the crown-ether macrocycle allows the Pe chromophores to adopt the most stable dimer configuration.

The effect of $\mathrm{Ba}^{2+}$ on the spectra can be explained by conformational changes of the $18 \mathrm{C6}$ host upon cation binding, ${ }^{\mathbf{5 3}, 59}$ as illustrated by representative snapshots in Fig. 2B. Along with the oxygen atoms of the crown-ether, the two amide oxygens are involved in metal binding and cause a rotation of the Pe sub-units leading to an increase of the Pe-Pe distance to $>6 \AA$ A. These simulations point to a broad distribution of geometries, especially in the presence of $\mathrm{Ba}^{2+}$, with distinct sub-populations, featuring T-shape and H-type conformers (snapshots b and c in Fig. 2B, respectively).

In contrast, the two-dimensional excitation/emission maps (Fig. S6 and S7†) suggest a narrowing of the distribution upon binding of a cation. This apparent contradiction with the MD simulations can be accounted for by the substantial increase of Pe-Pe distance upon complexation. Indeed, small structural changes at short distances, where the molecular orbitals of both sub-units overlap, have a stronger impact on the spectrum than large changes at long distances. This short-range sensitivity stems from CT-mediated exciton coupling $\left(J_{\mathrm{CT}}\right)$, which, in addition to the Coulombic interaction $\left(J_{\mathrm{C}}\right)$, predominates at close distances as it depends on the overlap of the frontier MOs of the two Pe sub-units. ${ }^{34}$ As shown in Fig. S9, $\dagger$ the flexible crown-ether backbone allows for a wide range of angles between the Pe long axes, altering $J_{\mathrm{CT}}$. On the other hand, if the Pe-Pe distance is too large for significant orbital overlap, the spectra are much less influenced by subtle conformational changes, explaining the excitation wavelength independent emission spectra of $18 \mathrm{C6} \subset \mathrm{Ba}^{2+}$.

\subsubsection{Time-resolved spectroscopy}

18C6. The early TA spectra measured with $18 \mathrm{C} 6$ in acetonitrile are dominated by a positive band peaking at around $700 \mathrm{~nm}$ and a negative band centred at about $420 \mathrm{~nm}$ (Fig. 3A). The positive band resembles the $\mathrm{S}_{n} \leftarrow \mathrm{S}_{1}$ absorption band of $\mathbf{P e},{ }^{\mathbf{6 0}, \mathbf{6 1}}$ but is significantly broader on the blue side. The negative band coincides with the stationary absorption spectrum and can be assigned to the ground-state bleach (GSB). No contribution from stimulated emission (SE) is observed. Within a few ps, the $700 \mathrm{~nm}$ band transforms into a band centred around $600 \mathrm{~nm}$ and, in parallel, a weak positive band rises around $1530 \mathrm{~nm}$ (Fig. 3B). Both bands decay concomitantly with the GSB on the tens of ns timescale (Fig. S16 $\dagger$ ).

These data were analysed globally assuming a series of four exponential steps and the resulting evolution associated difference spectra (EADS) and time constants are illustrated in Fig. 3C. These EADS and time constants cannot be systematically assigned to well-defined species/states and kinetic processes, respectively. Nevertheless, they give a semiquantitative picture of the spectral dynamics and of the timescales on which they occur. ${ }^{62}$

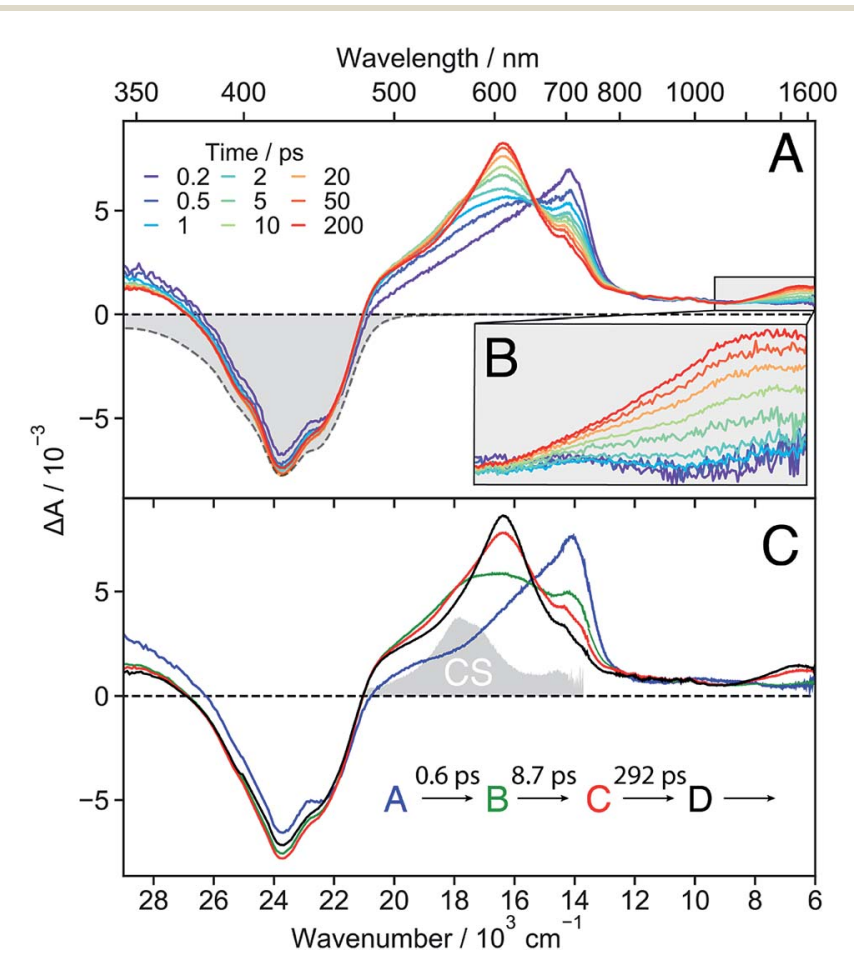

Fig. 3 (A) Transient absorption spectra measured at different time delays after excitation of $18 \mathrm{C} 6$ in acetonitrile together with the negative stationary absorption spectrum (grey). (B) Zoom in on the NIR region. (C) Evolution associated difference spectra (EADS) together with the corresponding time constants. The absorption spectrum of the charge-separated state measured with $18 \mathrm{C} 6 \subset \mathrm{Ba}^{2+}$ (vide infra) is depicted in grey to illustrate the spectral resemblance to the shoulder in EADS B and $C$. 
This analysis reveals that the transformation of the $700 \mathrm{~nm}$ band (EADS A) involves an intermediate stage, accounted for by EADS B and C, which are characterised by a shoulder at $560 \mathrm{~nm}$ and a weaker intensity than the long-lived $600 \mathrm{~nm}$ band (EADS D). The latter is very similar to the TA spectrum observed with highly concentrated Pe solutions in toluene and attributed to the excimer. ${ }^{63}$ Previous quantum-chemical calculations of the cofacially stacked Pe dimer predict that the equilibrium geometry of the lowest excitonic state, namely the excimer, ${ }^{64}$ adopts a $D_{2 \mathrm{~h}}$ geometry (Fig. S3 $\dagger$ ). During structural relaxation towards the excimer, the lowest energy absorption band shifts from the mid- to the near-IR region..$^{30,65}$ Based on this, the $1530 \mathrm{~nm}$ band can also be assigned to an intramolecular excimer. In the following, the term excimer will be strictly used to designate the relaxed $S_{1}$ excited state of the dimer. The 600 and $1530 \mathrm{~nm}$ excimer bands increase in intensity as the mutual orientation of the Pe heads evolves from the $D_{2}$ to the more stable $D_{2 \mathrm{~h}}$ geometry. The MD simulations indicate that the macrocycle allows for the eclipsed $D_{2 \mathrm{~h}}$ conformation, although it is not the most stable ground-state structure. Similar NIR excimer bands have been reported for perylene and perylenediimide dimers. ${ }^{\mathbf{5 1 , 6 6}}$

The $560 \mathrm{~nm}$ shoulder in EADS B and C is not visible in the medium and low polarity solvents, dichloromethane (DCM) and toluene (Fig. S17 $\dagger$ ). Moreover, it coincides with the absorption band of the CS state (vide infra) with the Pe radical cation and anion absorption bands at 540 and $580 \mathrm{~nm}$, respectively (grey in Fig. 3C). ${ }^{42,61,67}$ As this feature is not very pronounced and the coupling between the two Pe moieties, hence their molecular orbital overlap, is large, we attribute it to a SB-CT state rather than to a SB-CS state. Hereafter, we will call CS state, a state with clear spectral signature of ions, although this does not always imply a full CT character.

Given the distribution of conformations, a fraction of the excited-state population might directly form the excimer without going through the CT state. This appears to be the dominant pathway in the less polar solvents. Such distribution of geometries can also explain why features of both the CT and the excimer states are visible in EADS C.

The absence of SE in the early TA spectra suggests that these spectra (or EADS A) are not those of the initially photopopulated state, because excitation is done at a transition with a large dipole moment. This points to an ultrafast conversion from the initially populated bright state to a quasidark state, which equilibrates to the weakly emissive excimer. To unravel the processes occurring prior to the excimer formation, we applied time-resolved fluorescence spectroscopy, which has the advantage of emphasizing the excited states with a large emission dipole moment. As shown in Fig. 4, the timeresolved emission spectra differ significantly from the stationary spectrum. The early fluorescence band peaks at around $490 \mathrm{~nm}$ and its area is smaller by about one order of magnitude compared to that of the LE emission recorded under the same experimental conditions (vide infra), indicative of a significantly smaller transition dipole moment. This is fully consistent with the absence of a SE band in the TA spectra (Fig. 3). The $490 \mathrm{~nm}$ band transforms on similar timescales as

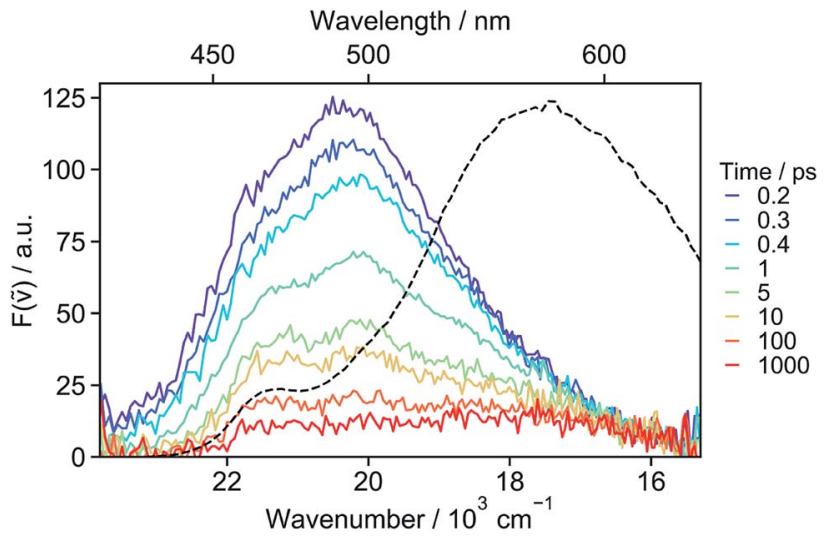

Fig. 4 Fluorescence spectra recorded at various time delays after $400 \mathrm{~nm}$ excitation of $18 \mathrm{C} 6$ in acetonitrile and stationary emission spectrum (dotted line).

the $\mathrm{A} \rightarrow \mathrm{B} \rightarrow \mathrm{C}$ steps in the TA data to a hardly visible band resembling the stationary excimer spectrum. As the groundstate population does not recover during this time interval, the decay of the fluorescence intensity can be interpreted as a decrease of the emission dipole moment. In the weakly polar toluene, the fluorescence decay is comparatively slower than in ACN (Fig. S24†). This can be explained by the fact that SB-CT accelerates the decrease of the oscillator strength in ACN, but does not prevent the formation of the excimer. SB occurs on the timescale of solvent fluctuations, whereas structural relaxation is comparatively slower. ${ }^{68-71}$

The ensemble of experimental results suggests that photoexcitation of $18 \mathrm{C} 6$ leads to the population of a higher excitonic state with a large oscillator strength (Fig. 5 , left). It is followed by an internal conversion to the lowest excitonic state, $S_{1}$, that is too fast to be resolved experimentally ( $\tau<100 \mathrm{fs}$ ). This ultrafast internal conversion prevents major geometrical reorganisation prior to population of the $S_{1}$ state, whose initial geometry is thus

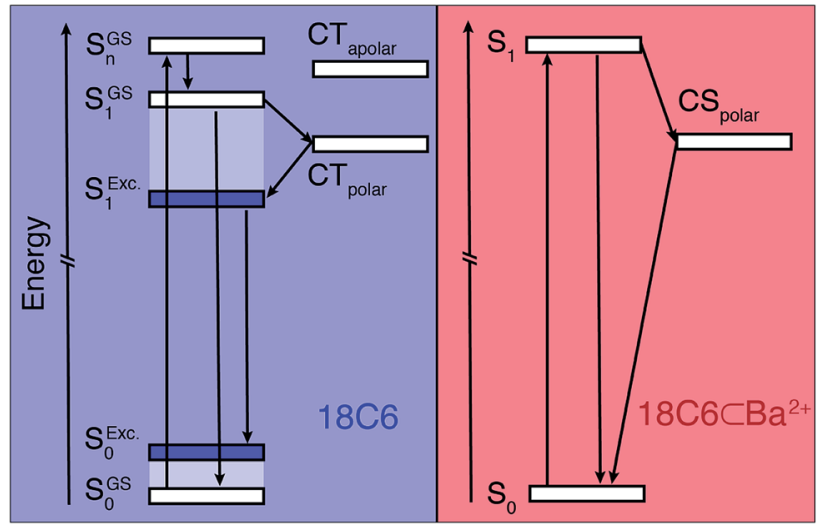

Fig. 5 Energy level diagrams illustrating the excited-state dynamics of $18 \mathrm{C} 6$ (left) and $18 \mathrm{C} 6 \subset \mathrm{Ba}^{2+}$ (right). The superscripts 'GS' and 'Exc.' stand for the ground-state equilibrium geometry and excited state equilibrium geometry (excimer), respectively. The $S$ states of $18 \mathrm{C} 6$ (left) are delocalised whereas that of the $18 \mathrm{C} 6 \subset \mathrm{Ba}^{2+}$ is mostly localised. 
similar to that in the ground state $\left(\mathrm{S}_{1}^{\mathrm{GS}}\right)$, i.e. mainly with a $D_{2}$ mutual orientation of the Pe sub-units. Fluctuations of the surrounding polar solvent leads to SB and confer a significant amount of CT character. However, as structural relaxation takes place, this state relaxes to the excimer equilibrium geometry, characterised by a lower CT character.

In toluene, SB-CT is not operative and the $\mathrm{S}_{1}$ state relaxes directly to the excimer. The excimer ultimately decays on a ns timescale (Fig. S16†), which is consistent with the large energy gap relative to the ground state and the vanishingly small emission transition dipole moment of a H-type dimer.

$18 \boldsymbol{C 6} \subset \boldsymbol{B a}^{2+}$. The TA spectra recorded at early time with $18 \mathrm{C6} \subset \mathrm{Ba}^{2+}$ exhibit a positive band around $700 \mathrm{~nm}$ and a negative band due to both GSB and SE (Fig. 6 and S15 $\dagger$ ). The spectra resemble those measured with Ref in DCM (Fig. S19†) as well as those reported for Pe. ${ }^{60,72}$ This implies that excitation is mostly localised on a single Pe sub-unit. Apart from small shifts of the SE and ESA bands attributed to vibrational relaxation, (Fig. S15 $\dagger)^{73-75}$ no significant dynamics occur during the first ns after excitation, in agreement with the weak coupling suggested by the stationary spectra and the MD simulations.

Both the $700 \mathrm{~nm}$ and SE bands decay on a few ns timescale in parallel with the rise of a band peaking at around $550 \mathrm{~nm}$ with a shoulder at $580 \mathrm{~nm}$. This band resembles the sum of the Pe radical cation and anion bands. ${ }^{42,61,67}$ It is much more pronounced than the shoulder observed with 18C6 in ACN (Fig. 3). Consequently, it is attributed to a SB-CS state, in agreement with the smaller coupling of the chromophores. It evolves in a few tens of ns to a very weak residual spectrum, that in turn decays on the $\mu$ s timescale. The last spectrum is similar to that of the triplet LE state of Pe. ${ }^{72,76}$

These data were analysed globally assuming a series of exponential steps and the resulting EADS and time constants are illustrated in the inset of Fig. 6. The A $\rightarrow$ B step can be attributed to the LE $\rightarrow$ CS process. A CS time constant of $25 \mathrm{~ns}$ is estimated from the $\mathrm{A} \rightarrow \mathrm{B}$ time constant and the fluorescence lifetime of Ref. This value, which should be considered as an average over a distribution of different conformers, points to

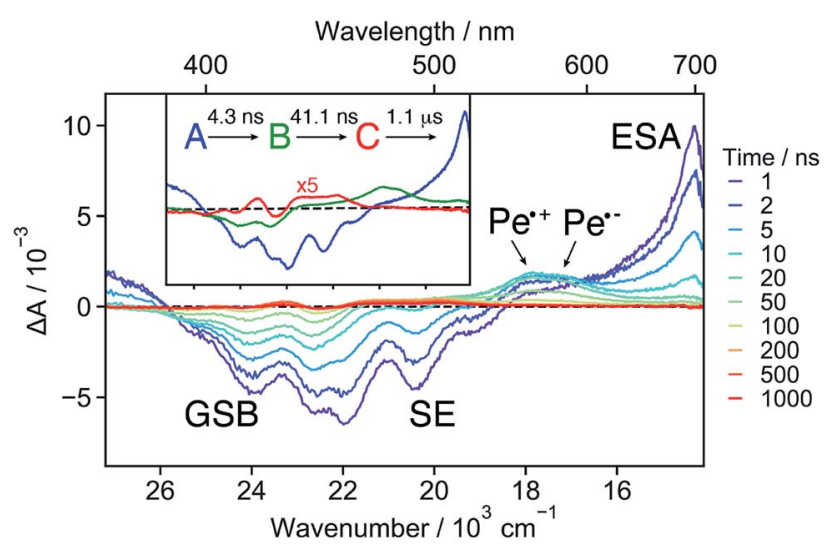

Fig. 6 Transient absorption spectra measured with $18 \mathrm{C} 6 \subset \mathrm{Ba}^{2+}$ in acetonitrile at different time delays after $355 \mathrm{~nm}$ excitation. The evolution associated difference spectra (EADS) obtained from a global analysis assuming a sequential model are shown in the inset. a $17 \%$ CS yield. Finally, the SB-CS state decays by charge recombination predominantly to the neutral ground state in about 40 ns.

SB-CS in $18 C 6 \subset \mathbf{B a}^{2+}$ is significantly slower than in the above-mentioned flexible Pe-Pr-Pe, with 12 and 130 ps time constants. ${ }^{42}$ The conformational space accessible to the 18C6 $\subset \mathrm{Ba}^{2+}$ is shifted towards larger distances compared to PePr-Pe in which the Pe sub-units can approach to less than $4 \AA$. The increased distance results in weaker electronic coupling and, thus, slower CS. Most probably, structural fluctuations are required for the subpopulations with the largest Pe-Pe distance for CS to be operative within the lifetime of the LE state.

The time-resolved emission spectra recorded with $18 \mathrm{C6} \subset \mathrm{Ba}^{2+}$ resemble the stationary spectrum (Fig. S23 $\dagger$ ). During the first ns after excitation, these spectra show relatively little dynamics, apart from some early band narrowing, due to vibrational relaxation already observed in the SE. ${ }^{74,75}$ The area is larger by approximately one order of magnitude compared to those measured with 18C6 (Fig. 4). This points to an emission from a LE state with a large transition dipole moment, in agreement with the presence of a SE band in the TA spectra.

The excited-state dynamics of $\mathbf{1 8 C 6} \subset \mathbf{B a}^{2+}$ are thus similar to those expected for two weakly coupled Pe chromophores in a polar environment. Initial population of a LE state is followed by SB-CS, in addition to the radiative and non-radiative relaxation to the ground state (Fig. 5 right).

\subsection{Searching for the "sweet spot"}

As shown above, the conformational restrictions imposed by the binding of $\mathrm{Ba}^{2+}$ to $18 \mathrm{C} 6$ render the eclipsed, close-distance $D_{2 \mathrm{~h}}$ excimer conformation inaccessible. Therefore, the SB-CS state becomes the lowest excited state in polar solvents. However, the slow electron transfer caused by the weak coupling between the chromophores leads to a CS yield of only $17 \%$. On the other hand, in the absence of cation, the $\pi$-stacked conformation undergoes ultrafast but incomplete SB-CS in polar solvents, and the excited state relaxes rapidly to the excimer.

Is there a "sweet spot" geometry between this two cases where fast and long-lived CS is possible? To answer this question, we investigated $18 \mathrm{C} 4$ and $16 \mathrm{C4}$, for which the conformational space accessible to the Pe chromophores differ from that of 18C6. Instead of $\mathrm{Ba}^{2+}$, which does not bind to all three crownethers, we used a $\mathrm{Na}^{+}$-salt $\left(\mathrm{NaBAr}_{\mathrm{F}}\right.$, sodium tetrakis[3,5bis(trifluoromethyl)phenyl]borate), which is soluble in the medium polarity solvent, DCM. For comparison, 18C6 was also studied in DCM with $\mathrm{Na}^{+}$.

3.2.1 Stationary spectra and MD simulations. The electronic spectra of $\mathbf{1 8 C 4}$ in DCM show indications of a strong coupling between the Pe moieties, similar to those observed with 18C6 (Fig. 7A). This is supported by the MD simulations, which predict the predominance of closely stacked conformations (Fig. 7B). The presence of LE-emission in $18 \mathrm{C6}$ but not 18C4 can be explained by the sub-populations with Pe-Pe distance $>6 \AA$, which is only significant for the former.

In contrast, the $I_{0-0} / I_{1-0}$ ratio in the absorption spectrum of the bichromophore host with the smallest crown-ether, 16C4, is 

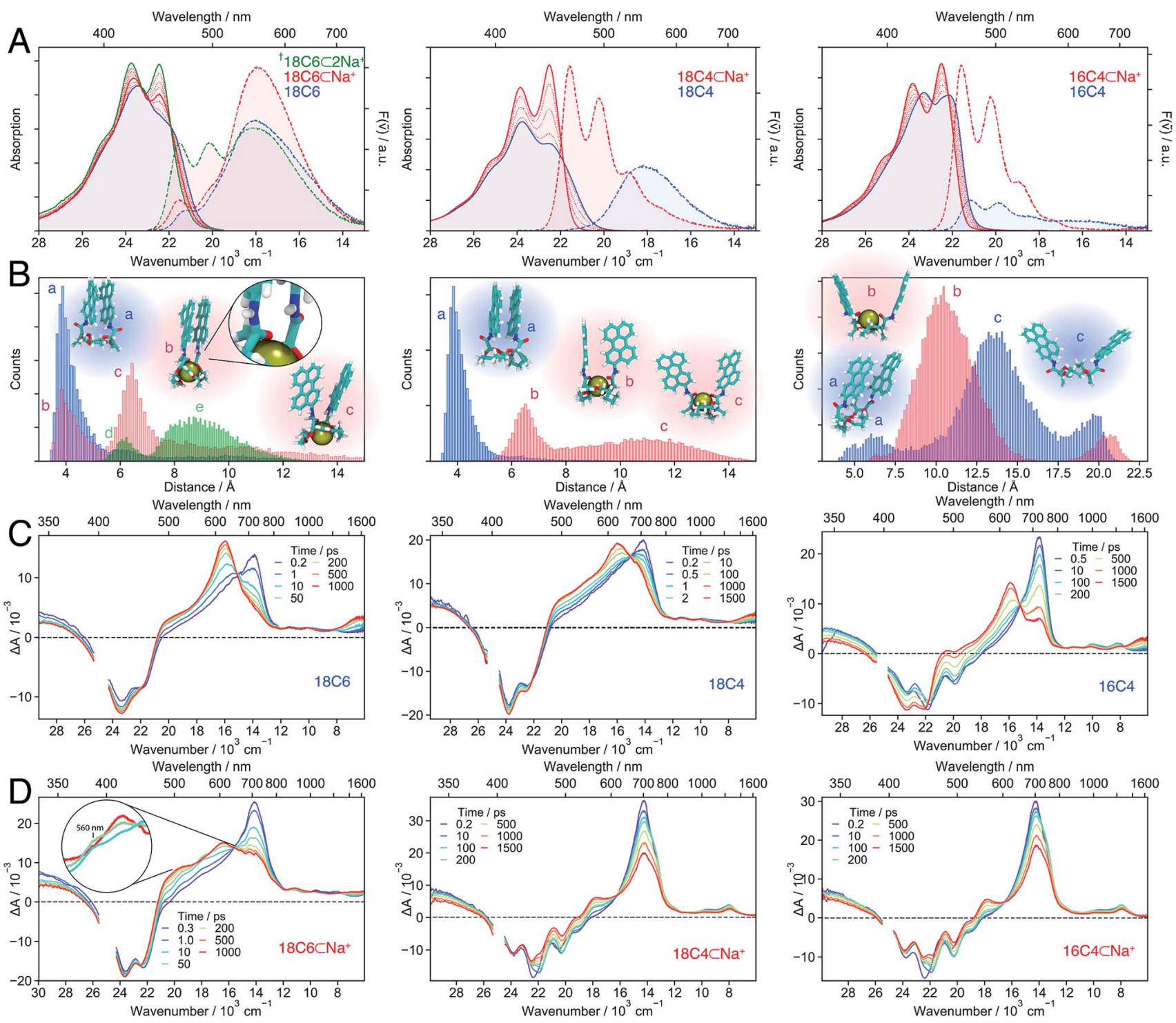

Fig. 7 Stationary electronic spectra (A), MD simulations (B) and transient absorption spectra of the hosts (C) and host-guest complexes (D) with $\mathrm{Na}^{+}$in DCM (left: 18C6; middle: 18C4; right: 16C4). The emission spectra can be quantitatively compared since the same solution and conditions were used and the changes in molar absorption coefficient at the excitation wavelength ( $400 \mathrm{~nm})$ upon cation binding do not change significantly. The $\dagger$ indicates that the spectra are due to a mixture of $1: 1$ and $1: 2$ complexes. The green histogram in the left panel of B corresponds to $18 \mathrm{C} 6 \subset 2 \mathrm{Na}^{+}$with the snapshots $d$ and e shown in Fig. 8B.

markedly larger and points to a weaker coupling relative to the other two macrocycles. Similarly, the fluorescence spectrum is dominated by the monomeric LE emission. The MD simulations suggest that $16 \mathrm{C} 4$ exhibits the broadest distribution of geometries and largest Pe-Pe distances. This is due to geometrical restrictions, which most likely hinder access towards the close-contact $D_{2}$ conformation, as reflected by the angle between the long axes of the Pe sub-units that deviates largely from $30^{\circ}$ (Fig. S13 $\uparrow$ ). The small difference in $I_{0-0} / I_{1-0}$ ratio between 16C4 and the monomeric Ref most likely originates from the small subpopulation with Pe-Pe distance of $<7.5 \AA$.

Upon addition of $\mathrm{Na}^{+}$, the vibronic structure of the absorption band of all three bichromophores becomes more pronounced. Moreover, the emission spectra of $18 \mathrm{C} 4$ and $16 \mathrm{C} 4$ are dominated by LE fluorescence. In the case of 18C6, the broad excimer-like band remains clearly visible even at the highest $\mathrm{Na}^{+}$concentration. Interestingly, the intensity of this band first increases with $\mathrm{Na}^{+}$concentration and then decreases.

The spectral changes measured with 18C4 and 16C4 could be reproduced by assuming $1: 1$ association model with the association constants and spectra given in Table S1 and Fig. S4. $\dagger$ MD simulations indicate that, upon complexation, the amide oxygens of the Pe chromophores, pointing originally towards the outside, bind to the cation. This requires a rotation of the amide groups, which in turn leads to the rotation of the whole Pe heads and to a concomitant increase of Pe-Pe distance. The simulations also reveal that complexation of 16C4 results in a decrease of the average Pe-Pe distance. Nevertheless, distances shorter than $7.5 \AA$ are geometrically unfavourable in $16 \mathrm{C4} \subset \mathrm{Na}^{+}$, and are only accessible in $16 \mathrm{C} 4$ 
alone. This supports the above hypothesis that the difference in $I_{0-0} / I_{1-0}$ ratio between $16 \mathrm{C} 4$ and Ref is only due to the small sub-population with Pe-Pe distance of $<7.5 \AA$.

In contrast, a $1: 2$ association model had to be assumed to reproduce the spectral changes measured with 18C6 upon addition of $\mathrm{Na}^{+}$. The $1: 1$ association constant is similar to those found for the other two crown-ethers (Table S1 $\dagger$ ), whereas the $1: 2$ association constant is smaller by three orders of magnitude. The binding of a single $\mathrm{Na}^{+}$to $18 \mathrm{C6}$ leads to a small increase of the $I_{0-0} / I_{1-0}$ ratio as well as a $\sim 50 \%$ increase of the excimer-like emission band. According to the MD simulations, only one of the Pe heads is rotated and its amide oxygen atom binds to the cation and to the $\mathrm{NH}$ group of the other chromophore (snapshot b in Fig. 7B left). Such asymmetric binding can be expected to result in dissimilar properties of the two Pe subunits and thus to an intrinsic SB. The magnitude of this effect was estimated by inspecting the changes in the stationary spectra of Ref upon addition of $\mathrm{Na}^{+}$. As shown in Fig. S5, $\dagger$ in the presence of $\mathrm{Na}^{+}$, both the absorption and emission bands of Ref shift to higher energy by about $500 \mathrm{~cm}^{-1}$ and the vibronic structure is more pronounced. As expected, the association constant is two orders of magnitude smaller than for the crownether hosts (Table S1 $\dagger$ ). The binding of $\mathrm{Na}^{+}$to the amide oxygen of Ref rises the energy of its first singlet excited state, most probably because the electron-donating ability of the amide substituent is switched off. ${ }^{77}$ As a consequence, Ref becomes similar to Pe upon complexation.

Even though full conversion to $18 \mathrm{C} 6 \subset 2 \mathbf{N a}^{+}$could not be achieved due to the limited solubility of $\mathrm{NaBAr}_{\mathrm{F}}$ in DCM, the absorption spectrum of $18 \mathrm{C6} \subset 2 \mathbf{N a}^{+}$could be obtained from the global analysis and resembles those of the $1: 1$ complexes of the two other crown-ethers (Fig. S4 $\dagger$ ). The $I_{0-0} / I_{1-0}$ ratio of the absorption spectrum and the presence of the monomeric LE emission point to a decrease of the coupling between the Pe moieties upon complexation of a second $\mathrm{Na}^{+}$. A stable $1: 2$ complex with two $\mathrm{Na}^{+}$was found in the MD simulations (Fig. 7B left), with two representative snapshots shown in Fig. 8A. One cation is bound to the crown-ether, whereas the other is symmetrically bound to the two amide oxygens. The second $\mathrm{Na}^{+}$ is possibly further stabilized by cation- $\pi$ interaction. ${ }^{78,79}$ Even though this interaction is predicted to be smaller if the cation is not centred above the $\pi$ surface, it can still have a stabilizing effect. ${ }^{80}$ The binding of a second cation leads to a Pe-Pe distance larger than $5.5 \AA$, in agreement with the smaller coupling inferred from the spectra.

Since the $1: 1$ and $1: 2$ association constants differ by three orders of magnitude, a symmetrically $\pi$-stacked conformer (18C6) and a $\pi$-stacked conformer with local asymmetry $\left(18 \mathrm{C6} \subset \mathrm{Na}^{+}\right)$can be quantitatively produced by adjusting the concentration of $\mathrm{NaBAr}_{\mathrm{F}}$ in DCM. Further addition of $\mathrm{Na}^{+}$leads to a mixture of $18 \mathrm{C6} \subset \mathbf{N a}^{+}$and $\mathbf{1 8 C 6} \subset \mathbf{2} \mathbf{N a}^{+}$. The excimer-like fluorescence measured at the highest $\mathrm{Na}^{+}$concentration is assigned to $18 \mathrm{C6} \subset \mathrm{Na}^{+}$and not to $18 \mathrm{C6} \subset 2 \mathrm{Na}^{+}$, as discussed in the ESI (Fig. S8†).

\subsubsection{Time-resolved spectroscopy}

Bichromophore hosts. The TA spectra measured with all three bichromophore hosts in DCM (Fig. 7C) confirm the different

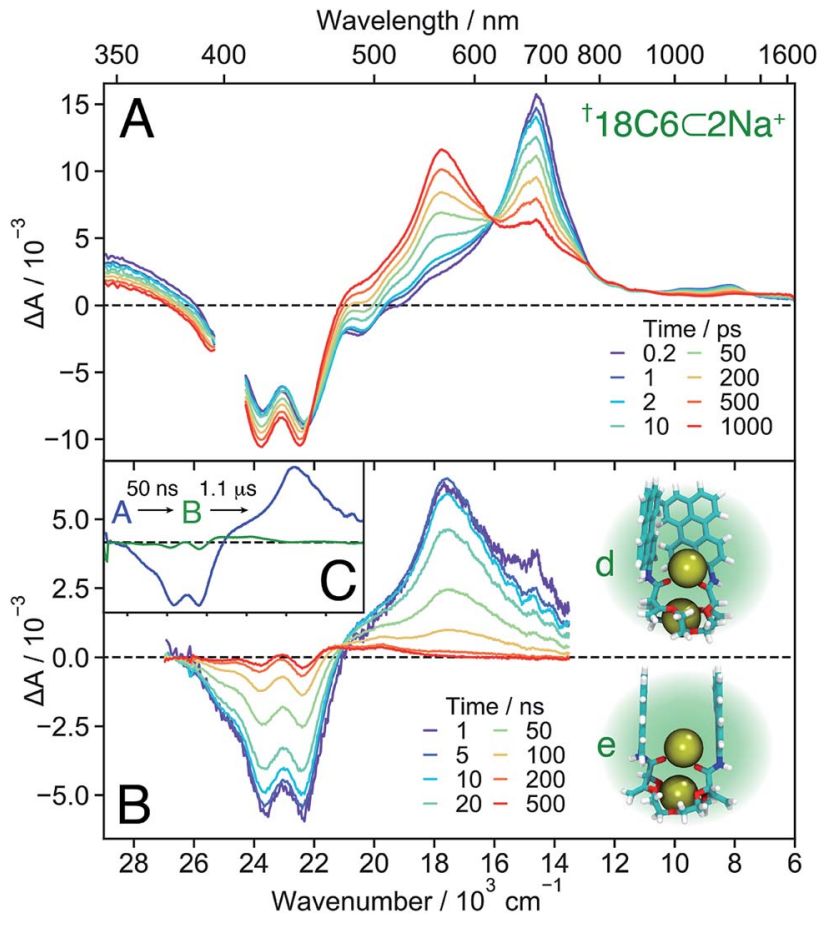

Fig. 8 Transient absorption spectra measured with $\dagger 18 \mathrm{C} 6 \subset 2 \mathrm{Na}^{+}$in $\mathrm{DCM}$ on the femtosecond $(\mathrm{A})$ and nanosecond $(\mathrm{B})$ timescale, together with snapshots obtained from MD simulations (see also Fig. 7B left). (C) Evolution associated difference spectra (EADS) obtained from a global analysis of the nanosecond transient absorption data.

extent of coupling revealed by the stationary spectra and $\mathrm{MD}$ simulations. No SE band can be detected in the $18 \mathrm{C} 6$ and $18 \mathrm{C} 4$ spectra, whereas it is clearly visible up to $1 \mathrm{~ns}$ in those recorded with 16C4. Like for 18C6 in ACN (Fig. 3), the spectra are first dominated by the $700 \mathrm{~nm}$ band and then show the build-up of the excimer bands around $600 \mathrm{~nm}$ and above $1500 \mathrm{~nm}$. However, the $560 \mathrm{~nm}$ shoulder, attributed to the SB-CT state, is no longer visible in the less polar DCM. The rise of the excimer bands occurs within a few tens of ps for $18 \mathrm{C} 6$ and $18 \mathrm{C} 4$ but is distinctly slower for $16 \mathrm{C} 4$, taking place on a $1 \mathrm{~ns}$ timescale. This difference is fully consistent with the MD simulations that predict much larger Pe-Pe distances in 16C4 than in the other two hosts. Thus, larger structural reorganisation is required to relax to the excimer geometry.

$1: 1$ Host-guest complexes. The TA spectra measured with both $18 \mathrm{C4} \subset \mathrm{Na}^{+}$and $16 \mathrm{C4} \subset \mathrm{Na}^{+}$in DCM exhibit a slow decay of the $700 \mathrm{~nm}$ and SE bands and a concomitant rise of a band around $560 \mathrm{~nm}$, that is attributed to a SB-CS state (Fig. 7D). The same $560 \mathrm{~nm}$ band is found a few tens of ns after excitation of Ref in DCM (Fig. S20 $\dagger$ ). It results from the self-quenching of Ref by SB-CS, as already observed with $\mathbf{P e} .^{81,82}$ The fact that the Pe cation and anion maxima cannot be distinguished here is probably due to the lower polarity of DCM compared to ACN. Another evidence for the assignment of this band to a SB-CS state of $18 \mathrm{C} 4 \subset \mathrm{Na}^{+}$and $16 \mathrm{C4} \subset \mathrm{Na}^{+}$rather than to an excimer is the absence of a NIR band.

The TA spectra measured with $18 \mathrm{C} 6 \subset \mathbf{N a}^{+}$in DCM show the very fast build up of a shoulder around $560 \mathrm{~nm}$, similar to that 
measured with the host alone in ACN (Fig. 3). We also attribute it to a CT rather than to a CS state, as it rapidly decays concomitantly with the rise of a band around $600 \mathrm{~nm}$. The latter differs significantly from the excimer band, and is not accompanied by a NIR band. This suggests that the relaxed excited state of $18 \mathrm{C6} \subset \mathrm{Na}^{+}$differs significantly from the relaxed excimer state observed with the 18C6 alone. This is further supported by the stationary fluorescence spectrum of $18 \mathrm{C6} \subset \mathrm{Na}^{+}$, which is more intense than that of 18C6, indicative of a stronger transition dipole moment. This state is tentatively interpreted as an excimer-like state with a geometry departing from that of the eclipsed $D_{2 \mathrm{~h}}$ excimer. Access to the latter geometry is probably hindered by the presence of $\mathrm{Na}^{+}$.

Occurrence of SB-CT for $18 \mathrm{C6} \subset \mathrm{Na}^{+}$in DCM, despite short Pe-Pe distances as in the host alone, could arise from the abovediscussed asymmetry caused by the binding of $\mathrm{Na}^{+}$to a single amide oxygen observed in the MD simulations. Without this intrinsic asymmetry, SB-CT in the medium polar DCM would be probably too slow to compete with the structural relaxation towards the excimer. The asymmetric binding of $\mathrm{Na}^{+}$and the ensuing differences in electronic structure of the two Pe heads could be a further reason why the relaxed excited state of the $18 \mathrm{C6} \subset \mathrm{Na}^{+}$complex differs spectrally from the excimer formed with the hosts alone.

$18 \mathrm{C6} \subset 2 \mathbf{N a}^{+}$. The TA spectra recorded with $18 \mathrm{C6}$ at the highest $\mathrm{Na}^{+}$concentration are presented in Fig. 8A. Based on the association constants and the molar absorption coefficients, the probability of the $1: 2$ complex to be excited at $400 \mathrm{~nm}$ is twice as large as that of the $1: 1$ complex. These spectra reveal that the build up of the $560 \mathrm{~nm}$ band is not followed by a rapid transformation to the $600 \mathrm{~nm}$ band as observed with the 1:1 complex, but decays to the ground state on a $50 \mathrm{~ns}$ timescale. This band is, thus, ascribed to a SB-CS state. The TA spectra shown in Fig. S18 † illustrates how the nature of the excited state of $18 \mathrm{C} 6$ and its dynamics change upon increasing $\mathrm{Na}^{+}$ concentration.

In contrast to all other conformers, SB-CS is nearly quantitative as indicated by the absence of ground-state recovery. The slight increase of the bleach is due to the decay of the overlapping $700 \mathrm{~nm}$ band and is expected for a nearly quantitative conversion. ${ }^{72}$ The high CS yield can be explained by a faster SBCS in $18 \mathrm{C6} \subset 2 \mathrm{Na}^{+}$compared to the $1: 1$ complexes of the other two macrocycles. This increased rate is assigned to the larger coupling between the Pe sub-units, as suggested by the MD simulations (Fig. 7B). They predict two main subpopulations: one with a $\sim 6 \AA$ Pe-Pe distance and a T-shape conformation, and one around 8-10 $\AA$ with a face-to-face conformation. The latter structure, with significant molecular orbital overlap and, thus, an enhanced CS rate, is not predicted for $18 \mathrm{C4} \subset \mathrm{Na}^{+}$and 16C4 $\subset \mathbf{N a}^{+}$. Indeed, the Pe-Pe distances in the face-to-face configurations are on average significantly larger in these two $1: 1$ complexes than in $18 \mathrm{C6} \subset \mathbf{2} \mathbf{N a}^{+}$. The second $\mathrm{Na}^{+}$interacts with both the amide oxygens and the $\pi$-surfaces of the two Pe heads and ensures a relatively close distance between the chromophores. The MD simulations indicate that, on average, the ions are located at equal distances from the two Pe heads but the difference in these distances fluctuates significantly
(Fig. S11†). These transient departures from symmetry due to the cations probably also contribute to the fast SB-CS. The cations should also participate to the stabilisation of the SB-CS state, additionally to the solvent. This stabilisation should mainly be due to Coulombic interactions and result in the translocation of the cation(s) towards the negatively-charged Pe head.

Finally, the second $\mathrm{Na}^{+}$also prevents further approach of the chromophores and excimer formation. Therefore, the CS state recombines relatively slowly to the ground state with a $50 \mathrm{~ns}$ time constant (Fig. 8B), very similar to that found for $18 \mathrm{C6} \subset \mathrm{Ba}^{2+}$ in ACN. The nearly "sweet spot" geometry, allowing fast and long-lived SB-CT, is realised with the $18 \mathrm{C6} \subset \mathbf{2} \mathbf{N a}^{+}$ complex.

\subsection{Structure-property relationship}

A comprehensive picture of the impact of structure on the nature of the excited state of Pe-based bichromophores can be drawn from this ensemble of results. Three main factors have to be considered: (i) the coupling between the two chromophores at the ground state geometry, (ii) their conformational flexibility, and (iii) their local environment, which includes the solvent and the cation(s). Their effect on the excited-state dynamics can be visualised by splitting the reaction coordinates into a structural and a solvation coordinate as depicted in Fig. 9. The structural coordinate describes both the Pe-Pe distance and their mutual orientation and is directly related to the coupling, increasing from left to right.

The extent of coupling at the ground state geometry (factor (i)) determines the initial position along this coordinate, whereas the conformational flexibility (factor (ii)) affects the capacity to evolve along this coordinate. Finally, the solvation coordinate plays a crucial role in the SB-CS process. Here, it includes the solvent polarisation, which depends on the solvent polarity and also the asymmetry in cation position in the complex (factor (iii)). The extent of CT character of the excited state increases along this coordinate.

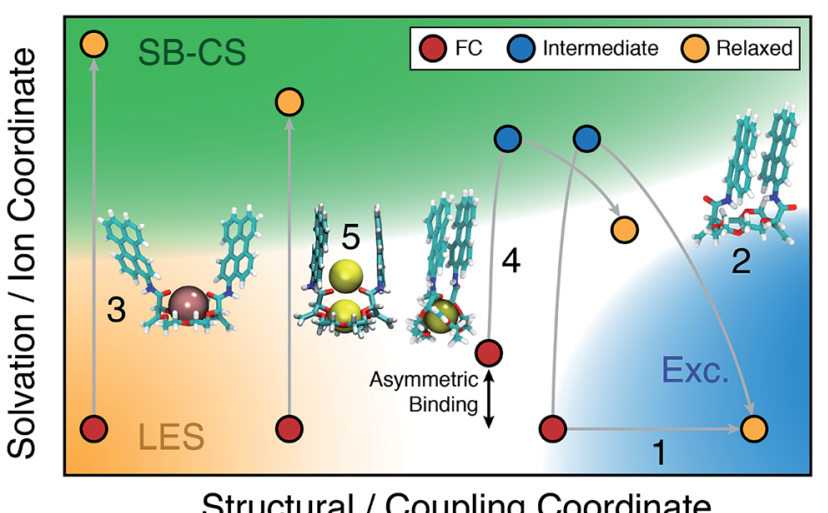

Fig. 9 Schematic two-dimensional representation of the relaxation pathways (gray lines) on the $S_{1}$ surface of the $18 C 6$ host, $1: 1$ and $1: 2$ complexes (see text for details). FC: initial location at the ground-state geometry; LES: locally excited state; Exc.: excimer. The coloured area refer to the nature of the excited state. 
For example, the excited-state dynamics of 18C6 in toluene can be represented by a simple evolution along the structural coordinate (trajectory 1 in Fig. 9). By contrast, in ACN, the system first moves along the faster solvation coordinate to an intermediate SB-CT state before evolving along the structural coordinate towards the more stable excimer state (trajectory 2). Polar solvation does not suffice to stabilise a significant CT character in a compact dimer. Thus, solvent relaxation around the weakly polar excimer corresponds to a 'backward motion' along the solvent coordinate. Upon complexation with $\mathrm{Ba}^{2+}$, the structural coordinate is essentially frozen at a weak coupling position and the reaction can only proceed along the solvent coordinate towards the SB-CS state (trajectory 3). Replacement of $\mathrm{Ba}^{2+}$ by $\mathrm{Na}^{+}$and ACN by DCM, leads to trajectory 4 where the relaxed state should have some excimer character as well as some asymmetry due to the presence of the cation.

Finally, in the "sweet spot" geometry, the structural coordinate should be locked in an intermediate position, where coupling is large enough to ensure fast SB-CS, but sufficiently small to prevent the collapse to an excimer-like state. This condition seems to be fulfilled for $18 \mathrm{C6} \subset \mathbf{2} \mathbf{N a}^{+}$(trajectory 5 ). In DCM, the evolution along the solvation coordinate is limited but is compensated by the presence of the ions. If the interactions with the ions are not the same for the two Pe heads, the latter can no longer be considered as two identical chromophores. This leads to an intrinsic SB and favours CS in one direction with respect to the other. In the absence of ions, symmetry breaking is only driven by the thermal fluctuations of the solvent field, that transiently lifts the degeneracy of the two CS states. Consequently, the increase of the driving force arising from such asymmetric ion-chromophore interactions plays in favour of a faster SB-CS.

The results obtained with the other two bichromophores can be rationalised in a similar way. The hosts alone follow trajectories that involve mostly the structural coordinate (trajectory 1 ), whereas the trajectories of the $1: 1$ complexes are close to trajectory 3 .

\section{Conclusions}

These crown-ether based Pe dimers allowed establishing a comprehensive structure/property relationship. Given their flexibility, MD simulations proved to be crucial for rationalising the spectroscopic observations. The results presented here demonstrate that these bichromophores allow the coupling to be varied in a relatively well-controlled fashion over a wide range, contrary to previous studies. This is achieved by first changing the size and nature of the macrocycle. However, as all three crown-ethers allow for a relatively large conformational flexibility, the excited bichromophores finally equilibrate to an intramolecular excimer inhibiting the population of the CS state.

The second way to modify the coupling is upon complexation with one or two cations. Cation binding not only changes the ground-state geometry and decreases the coupling, but also prevents the close approach of the Pe heads and, thus, excimer formation. As a consequence, the excited state relaxes to a SB-CS state. The exception is $\mathbf{1 8 C 6} \subset \mathbf{N a}^{+}$where close Pe-Pe distances are still accessible.

Our results also suggest that symmetry breaking in the complexes is facilitated by the imbalanced interactions of the ion(s) with the two Pe heads. Because of this, the two chromophores are not strictly identical and SB-CS is no longer only controlled by solvent fluctuations.

All these effects play advantageously in the "sweet spot" geometry to achieve quantitative $\mathrm{CS}$ with a relatively long lifetime.

The structure/property relationship found here with Pe dimers, can be expected to be largely valid for the other dyes that are often used as building blocks in multichromophoric architectures. Therefore, these principles and quantitative correlations could be used advantageously for optimising the CS properties of such systems.

\section{Conflicts of interest}

There are no conflicts to declare.

\section{Acknowledgements}

Financial support from the National Institutes of Health (grant P41-GM104601 (E. T)), the 'Fonds National Suisse de la Recherche Scientifique' (grants PZ00P2_174116 (T. K.), 200020184843 (J. L.) and 200020-184607 (E. V.)) and the University of Geneva is acknowledged. Luc Monnier is thanked for his participation to the synthesis of compounds $18 \mathrm{C} 4$ and 16C4. The simulations were performed using resources provided by XSEDE (grant MCA06N060) and NCSA Blue Waters.

\section{Notes and references}

$\$$ CS will be indistinctly used as an abbreviation for 'charge separation' and 'charge separated'.

1 V. Sundström, T. Pullerits and R. van Grondelle, J. Phys. Chem. B, 1999, 103, 2327-2346.

2 G. Kodis, Y. Terazono, P. A. Liddell, J. Andréasson, V. Garg, M. Hambourger, T. A. Moore, A. L. Moore and D. Gust, J. Am. Chem. Soc., 2006, 128, 1818-1827.

3 M. R. Wasielewski, J. Org. Chem., 2006, 71, 5051-5066.

4 I. Oesterling and K. Muellen, J. Am. Chem. Soc., 2007, 129, 4595-4605.

5 V. Sundström, Annu. Rev. Phys. Chem., 2008, 59, 53-77.

6 N. Aratani, D. Kim and A. Osuka, Acc. Chem. Res., 2009, 42, 1922-1934.

7 Y. C. Cheng and G. R. Fleming, Annu. Rev. Phys. Chem., 2009, 60, 241.

8 G. Bottari, G. de la Torre, D. M. Guldi and T. Torres, Chem. Rev., 2010, 110, 6768-6816.

9 M. C. O'Sullivan, J. K. Sprafke, D. V. Kondratuk, C. Rinfray, T. D. W. Claridge, A. Saywell, M. O. Blunt, J. N. O'Shea, P. H. Beton, M. Malfois and H. L. Anderson, Nature, 2011, 469, 72-75. 
10 G. D. Scholes, G. R. Fleming, A. Olaya-Castro and R. van Grondelle, Nat. Chem., 2011, 3, 763.

11 M. F. Hohmann-Marriott and R. E. Blankenship, Annu. Rev. Plant Biol., 2011, 62, 515-548.

12 G. de la Torre, G. Bottari, M. Sekita, A. Hausmann, D. M. Guldi and T. Torres, Chem. Soc. Rev., 2013, 42, 80498105.

13 R. Ziessel, G. Ulrich, A. Haefele and A. Harriman, J. Am. Chem. Soc., 2013, 135, 11330-11344.

14 H.-Q. Peng, L.-Y. Niu, Y.-Z. Chen, L.-Z. Wu, C.-H. Tung and Q.-Z. Yang, Chem. Rev., 2015, 115, 7502-7542.

15 A. Chenu and G. D. Scholes, Annu. Rev. Phys. Chem., 2015, 66, 69-96.

16 L. Hammarström, Acc. Chem. Res., 2015, 48, 840-850.

17 D. Gust, T. A. Moore and A. L. Moore, Acc. Chem. Res., 2001, 34, 40-48.

18 D. Holten, D. F. Bocian and J. S. Lindsey, Acc. Chem. Res., 2002, 35, 57-69.

19 A. Morandeira, E. Vauthey, A. Schuwey and A. Gossauer, J. Phys. Chem. A, 2004, 108, 5741-5751.

20 M. R. Wasielewski, Acc. Chem. Res., 2009, 42, 1910-1921.

21 T. Weil, T. Vosch, J. Hofkens, K. Peneva and K. Müllen, Angew. Chem., Int. Ed., 2010, 49, 9068-9093.

22 R. Bhosale, J. Míšek, N. Sakai and S. Matile, Chem. Soc. Rev., 2009, 39, 138-149.

23 Y. Terazono, G. Kodis, M. Chachisvilis, B. R. Cherry, M. Fournier, A. Moore, T. A. Moore and D. Gust, J. Am. Chem. Soc., 2014, 245-258.

24 L. Favereau, A. Makhal, Y. Pellegrin, E. Blart, J. Petersson, E. Göransson, L. Hammarström and F. Odobel, J. Am. Chem. Soc., 2016, 138, 3752-3760.

25 C. Kaufmann, W. Kim, A. Nowak-Król, Y. Hong, D. Kim and F. Würthner, J. Am. Chem. Soc., 2018, 140, 4253-4258.

26 K. Miyata, F. S. Conrad-Burton, F. L. Geyer and X. Y. Zhu, Chem. Rev., 2019, 119, 4261-4292.

27 F. C. Spano, Acc. Chem. Res., 2010, 43, 429-439.

28 N. J. Hestand and F. C. Spano, Acc. Chem. Res., 2017, 50, 341350.

29 M. Kasha, H. R. Rawls and E.-B. M. Ashraf, Pure Appl. Chem., 1965, 11, 371-392.

30 B. Engels and V. Engel, Phys. Chem. Chem. Phys., 2017, 19, 12604-12619.

31 A. Davydov, Theory of Molecular Excitons, Springer, US, 1971.

32 Y. Wu, J. Zhou, B. T. Phelan, C. M. Mauck, J. F. Stoddart, R. M. Young and M. R. Wasielewski, J. Am. Chem. Soc., 2017, 139, 14265-14276.

33 M. I. S. Röhr, H. Marciniak, J. Hoche, M. H. Schreck, H. Ceymann, R. Mitric and C. Lambert, J. Phys. Chem. C, 2018, 122, 8082-8093.

34 N. J. Hestand and F. C. Spano, Chem. Rev., 2018, 118, 70697163.

35 J. M. Giaimo, A. V. Gusev and M. R. Wasielewski, J. Am. Chem. Soc., 2002, 124, 8530-8531.

36 M. J. Fuller, A. V. Gusev and M. R. Wasielewski, Isr. J. Chem., 2004, 44, 101-108.

37 M. W. Holman, P. Yan, D. M. Adams, S. Westenhoff and C. Silva, J. Phys. Chem. A, 2005, 109, 8548-8552.
38 N. Banerji, A. Fürstenberg, S. Bhosale, A. L. Sisson, N. Sakai, S. Matile and E. Vauthey, J. Phys. Chem. B, 2008, 112, 89128922.

39 N. Banerji, G. Duvanel, A. Perez-Velasco, M. Santanu, N. Sakai, S. Matile and E. Vauthey, J. Phys. Chem. A, 2009, 113, 8202-8212.

40 N. Sakai, M. Lista, O. Kel, S.-i. Sakurai, D. Emery, J. Mareda, E. Vauthey and S. Matile, J. Am. Chem. Soc., 2011, 133, 1522415227.

41 A. N. Bartynski, M. Gruber, S. Das, S. Rangan, S. Mollinger, C. Trinh, S. E. Bradforth, K. Vandewal, A. Salleo, R. A. Bartynski, W. Bruetting and M. E. Thompson, J. Am. Chem. Soc., 2015, 137, 5397-5405.

42 V. Markovic, D. Villamaina, I. Barabanov, L. M. Lawson Daku and E. Vauthey, Angew. Chem., Int. Ed., 2011, 50, 7596-7598. 43 E. Vauthey, ChemPhysChem, 2012, 13, 2001-2011.

44 S. Das, W. G. Thornbury, A. N. Bartynski, M. E. Thompson and S. E. Bradforth, J. Phys. Chem. Lett., 2018, 9, 3264-3270.

45 N. Mataga, H. Yao, T. Okada and W. Rettig, J. Phys. Chem., 1989, 93, 3383-3386.

46 B. Dereka, M. Koch and E. Vauthey, Acc. Chem. Res., 2017, 50, 426-434.

47 R. J. Lindquist, K. M. Lefler, K. E. Brown, S. M. Dyar, E. A. Margulies, R. M. Young and M. R. Wasielewski, J. Am. Chem. Soc., 2014, 136, 14912-14923.

48 Y. Wu, R. M. Young, M. Frasconi, S. T. Schneebeli, P. Spenst, D. M. Gardner, K. E. Brown, F. Würthner, J. F. Stoddart and M. R. Wasielewski, J. Am. Chem. Soc., 2015, 137, 1323613239.

49 M. Son, K. H. Park, C. Shao, F. Würthner and D. Kim, J. Phys. Chem. Lett., 2014, 5, 3601-3607.

50 F. Würthner, C. R. Saha-Möller, B. Fimmel, S. Ogi, P. Leowanawat and D. Schmidt, Chem. Rev., 2016, 116, 962-1052.

51 R. E. Cook, B. T. Phelan, R. J. Kamire, M. B. Majewski, R. M. Young and M. R. Wasielewski, J. Phys. Chem. A, 2017, 121, 1607-1615.

52 A. Homberg, E. Brun, F. Zinna, S. Pascal, M. Górecki, L. Monnier, C. Besnard, G. Pescitelli, L. D. Bari and J. Lacour, Chem. Sci., 2018, 9, 7043-7052.

53 M. Vishe, T. Lathion, S. Pascal, O. Yushchenko, A. Homberg, E. Brun, E. Vauthey, C. Piguet and J. Lacour, Helv. Chim. Acta, 2018, 101, e1700265.

54 F. Zinna, S. Voci, L. Arrico, E. Brun, A. Homberg, L. Bouffier, T. Funaioli, J. Lacour, N. Sojic and L. Di Bari, Angew. Chem., Int. Ed., 2019, 58, 6952-6956.

55 Z. Jarolímová, M. Vishe, J. Lacour and E. Bakker, Chem. Sci., 2015, 7, 525-533.

56 M. Vishe, R. Hrdina, A. I. Poblador-Bahamonde, C. Besnard, L. Guénée, T. Bürgi and J. Lacour, Chem. Sci., 2015, 6, 49234928.

57 P. C. Johnson and H. W. Offen, Chem. Phys. Lett., 1973, 18, 258-260.

58 K. Nakagawa, Y. Numata, H. Ishino, D. Tanaka, T. Kobayashi and E. Tokunaga, J. Phys. Chem. A, 2013, 117, 11449-11455.

59 C. J. Pedersen, J. Am. Chem. Soc., 1967, 89, 7017-7036. 
60 C. R. Goldschmidt and M. Ottolenghi, J. Phys. Chem., 1971, 75, 3894-3897.

61 N. Mataga, T. Asahi, Y. Kanda, T. Okada and T. Kakitani, Chem. Phys., 1988, 127, 249-261.

62 I. H. M. van Stokkum, D. S. Larsen and R. van Grondelle, Biochim. Biophys. Acta, Bioenerg., 2004, 1657, 82-104.

63 R. Katoh, S. Sinha, S. Murata and M. Tachiya, J. Photochem. Photobiol., A, 2001, 145, 23-34.

64 R. D. Pensack, R. J. Ashmore, A. L. Paoletta and G. D. Scholes, J. Phys. Chem. C, 2018, 122, 21004-21017.

65 D. Casanova, Int. J. Quantum Chem., 2015, 115, 442-452.

66 K. E. Brown, W. A. Salamant, L. E. Shoer, R. M. Young and M. R. Wasielewski, J. Phys. Chem. Lett., 2014, 5, 2588-2593. 67 T. Shida and S. Iwata, J. Am. Chem. Soc., 1973, 95, 3473.

68 K. A. Zachariasse, G. Duveneck and R. Busse, J. Am. Chem. Soc., 1984, 106, 1045-1051.

69 F. D. Lewis, G. D. Reddy, D. M. Bassani, S. Schneider and M. Gahr, J. Am. Chem. Soc., 1994, 116, 597-605.

70 R. M. Stratt and M. Maroncelli, J. Phys. Chem., 1996, 100, 12981-12996.

71 T. Kumpulainen, B. Lang, A. Rosspeintner and E. Vauthey, Chem. Rev., 2017, 117, 10826-10939.
72 G. Angulo, A. Rosspeintner, B. Lang and E. Vauthey, Phys. Chem. Chem. Phys., 2018, 20, 25531-25546.

73 C. Rulliere, A. Declemy and P. Kottis, Chem. Phys. Lett., 1984, 110, 308-314.

74 T. Kasajima, S. Akimoto, S.-I. Sato and I. Yamazaki, J. Phys. Chem. A, 2004, 108, 3268.

75 A. Pigliucci, G. Duvanel, L. M. L. Daku and E. Vauthey, J. Phys. Chem. A, 2007, 111, 6135-6145.

76 F. Lewitzka and H.-G. Löhmannsröben, Z. Phys. Chem., 1990, 169, 203-218.

77 O. F. Mohammed, J. Phys. Chem. A, 2010, 114, 11576-11582.

78 D. A. Dougherty, Acc. Chem. Res., 2013, 46, 885-893.

79 A. S. Mahadevi and G. N. Sastry, Chem. Rev., 2013, 113, 21002138.

80 M. S. Marshall, R. P. Steele, K. S. Thanthiriwatte and C. D. Sherrill, J. Phys. Chem. A, 2009, 113, 13628-13632.

81 K. H. Grellmann and A. R. Watkins, Chem. Phys. Lett., 1971, 9, 439-443.

82 E. Vauthey, P. Suppan, E. Haselbach and R. S. Davidson, Helv. Chim. Acta, 1986, 69, 430-437. 